\title{
Current
}

\section{Helping older adults overcome the challenges of technology}

\section{Interventions need to focus on both cognitive enhancement and functional skills training}

$\mathrm{T}$ echnology is pervasive, and for many people, it is central to their daily activities. Younger people who have been exposed to technology for their entire lives take this for granted, but older individuals often have had much less experience with it. Many technological developments that are now a part of most people's daily life, such as personal computers, cell phones, and automated teller machines (ATMs), have occurred in the past 4 decades, with the pace accelerating in the last 15 to 20 years.

Such changes have had a substantial impact on older adults who were never exposed to these technologies during their working life. For example, an 85-year-old person who retired at age 65 would probably have not been exposed to wireless internet prior to retirement. Therefore, all of the tasks that they are now required to complete online would have been performed in other ways. Banking, accessing instruction manuals for new devices, and even scheduling and confirming health care appointments and accessing medical records all now require individuals to have a level of technological skills that many older individuals find challenging. At times, this can limit their ability to complete routine daily activities, and also can have clinical implications (Table, page 14).

continued

\section{Disclosures}

Dr. Harvey has received consulting fees or travel reimbursements from Alkermes, Bio Excel, Boehringer Ingelheim, Intra-Cellular Therapies, Mindstrong Health, Minerva Pharma, Regeneron Pharma, Roche Pharma, Sunovion Pharma, Takeda Pharma, and Teva. He receives royalties from the Brief Assessment of Cognition in Schizophrenia. He is Chief Scientific Officer of i-Function, Inc. He has research grants from Takeda and the Stanley Medical Research Foundation. Dr. Nascimento reports no financial relationships with any companies whose products are mentioned in this article, or with manufacturers of competing products.

doi: $10.12788 /$ cp. 0021

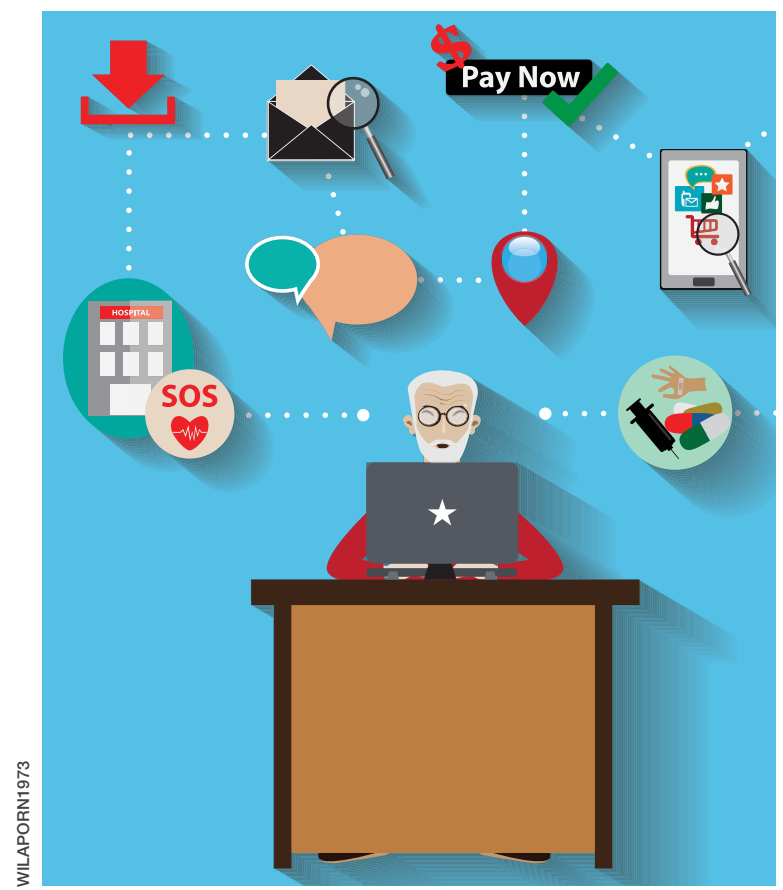

Philip D. Harvey, PhD

Leonard M. Miller Professor of Psychiatry and Behavioral Sciences

Department of Psychiatry and Behavioral Sciences University of Miami Miller School of Medicine Miami, Florida

\section{Vanessa Nascimento, MD, MPH}

PGY-1 Psychiatry Resident

University of Miami/Jackson Health System Psychiatry Residency Training Program

Department of Psychiatry and Behavioral Sciences

University of Miami Miller School of Medicine

Miami, Florida 


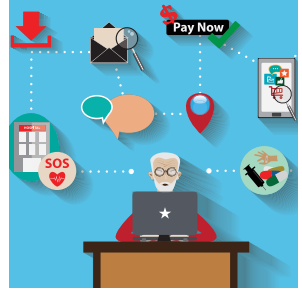

Older adults and technology

\section{Clinical Point}

Technological skills differ from other skills due to the constant updating of devices, programs, and applications

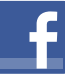

Discuss this article at www.facebook.com/ MDedgePsychiatry

\section{Table}

\section{Older adults and technological skills: Clinical implications}

Learning new technology-based skills is challenging for healthy older people

Individuals who have the greatest challenges learning technology-related skills are commonly those who have the least opportunity to avoid using technology

These technology-based challenges can interfere with medical and psychiatric treatments, because technology may be required to schedule or confirm appointments, fill prescriptions, or access medical records

Clinicians should not assume that their patients have learned the skills required to actively participate in health care that requires the use of technology

Treatment for normal age-related cognitive changes is available with computerized cognitive training

Technology-based skills need to be taught separately from interventions that focus on cognitive enhancement

Fortunately, there are strategies clinicians can use to help their older patients face these challenges. In this article, we describe the cognitive domains associated with learning technological skills, how aging affects these domains, and what can be done to help older adults improve their technological skills.

\section{Limited training on how to use new technology}

Technological skills are similar to any other skills in one critical way: they need to be learned. At the same time, technological skills also differ from many other skills, such as playing a musical instrument, because of the constant updating of devices, programs, and applications. When smartphones or computers update their operating systems, the visual appearance of the screen and the way that tasks are performed also can change. Buttons can move and sequences of commands can be altered. Updates often happen with little or no notice, and users may need to navigate a completely different device landscape in order to perform tasks that they had previously mastered.

In addition, the creators/distributors of technology typically provide little training or documentation. Further, institutions such as banks or health care systems frequently do not provide any specific training for using their systems. For example, when patients are required to use technology to refill prescriptions, typically there is no training available on how the system operates.

\section{Cognitive domains associated with technological skills}

Because there are minimal opportunities to receive training in how to use most aspects of technology, users have to be able to learn by exposure and experience. This requires several different cognitive abilities to work together. In a recent review, Harvey ${ }^{1}$ described cognition and cognitive assessment in the general population, with a focus on cognitive domains. Here we discuss several of these domains in terms of the relationship to real-world functional tasks and discuss their importance for mastering technology.

Reasoning and problem solving. Because most technological devices and applications are designed to be "intuitive," the user needs to be able to adopt a sequential approach to learning the task. For example, using the internet to refill a prescription requires several steps:

- accessing the internet

- finding the pharmacy web site

- establishing a user ID and password

- navigating the web site to the prescriptions section

- identifying the correct prescription

- requesting the refill

- selecting the pickup date and time. After navigating these steps, an individual still needs other cognitive abilities to refill other prescriptions later. However, executive functioning is also critical for maintaining organization across different technological demands. For example, web sites have different password rules and require frequent changes without reusing old passwords, so it becomes critical 
to maintain an organized list of web site addresses and their passwords.

Refilling a prescription with a telephone voice menu also requires a series of steps. Typically, this process is simpler than an internet refill, because no log-in information is necessary. However, it still requires a structured series of tasks.

Working memory refers to the ability to hold information in consciousness long enough to operate on it. At each step of the navigation process, the user needs to remember which steps he/she has already completed, because repeating steps can slow down the process or lead to error messages. Thus, remembering which steps have been completed is as critical for performing tasks as is correctly understanding the anticipated sequence of steps. Further, when a password is forgotten, the user needs to remember the newly provided password.

Working memory can be spatial as well. For example, most web sites do not display a password while it is being entered, which eliminates spatial working memory from the equation. Thus, the ability to remember which characters have been entered and which still need to be entered is necessary.

Episodic memory is the process of learning and retaining newly presented verbal or spatial information as well as recalling it later for adaptive use. After successfully using a new technology, it is critical to be able to remember what to do the next time it is used. This includes both recalling how to access the technology (including the web address, user ID, and password), recalling the steps needed to be performed and their sequence, and recognizing the buttons and instructions presented onscreen.

Procedural memory is memory for motor acts and sequences. For instance, remembering how to ride a bicycle is a procedural memory, as is the ability to perform motor acts in sequence, such as peeling, cutting, and cooking vegetables. Interestingly, procedural memory can be spared in individuals with major challenges in episodic memory, such as those with amnestic conditions or cortical dementia. Thus, it may be possible for people to continue to perform technology-based skills despite declines in episodic memory. Many current technological functional tasks have fixed sequences of events that, if remembered, can lead to increased efficiency and higher chances of success in performance of functional tasks.

Prospective memory is the ability to remember to perform tasks in the future. This can include event-related tasks (eg, enter your password before trying to make a hotel reservation on a web site) or timerelated tasks (eg, refill your prescriptions next Friday). Technology can actually facilitate prospective memory by providing reminders to individuals, such as alarms for appointments. However, prospective memory is required to initially set up such alarms, and setting up confusing or incorrect alarms can impede task performance.

Processing speed is the ability to perform cognitively demanding tasks under time constraints. Traditional processing speed tasks include coding and sorting tasks, which require processing new information and effort for relatively short periods of time. In our research, we discovered that processing speed measured with traditional tests was strongly correlated with the time required to perform functional tasks such as an ATM banking task. ${ }^{2,3}$ This correlation makes sense in terms of the fact that many real-world functional tasks with technology often have a series of sequential demands that must be accomplished before progression to the next task.

Manual dexterity is also important for using technology. Many electronic devices have small, touch screen-based keyboards. Being able to touch the correct key requires dexterity and can be made more difficult by age-related vision changes, a tremor, or reduced sensation in extremities.

\section{Cognitive changes and aging}

It is normal for certain cognitive abilities to change with aging. There are a set of cognitive skills that are generally stable from early adulthood until the early "senescent" period. Some of these skills decline normatively after age 60 to 65 , or earlier in some individuals. These include processing new information, solving new problems, and

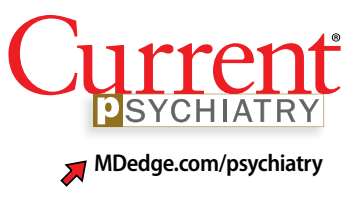

Clinical Point

Technology can facilitate prospective memory by providing reminders such as alarms for appointments 


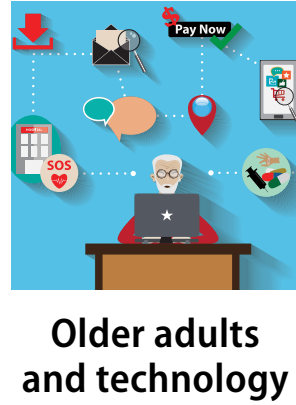

Clinical Point

Learning how to use new technologies requires fluid intelligence, which is less efficient in older adults

\section{Box}

\section{The aging brain's effects on cognitive function}

1 he global baseline intensity of human brain activity, determined by indirectly measuring blood oxygenation, decreases with age. ${ }^{10}$ Multiple domains of fluid cognition decline with age; these cognitive abilities include processing speed, ${ }^{11,12}$ working memory, ${ }^{11}$ episodic memory, ${ }^{11}$ and executive function. ${ }^{11}$ Expected neuroanatomic changes of aging include a decrease in cerebral grey matter volume as well as decreased white matter integrity, which is associated with diminished executive function and impaired working memory..$^{13}$ Processing speed is associated with increased white matter microstructure during neurodevelopment. ${ }^{14}$ Diminished processing speed in older adults also may predict increased mortality risk..$^{15}$ Individuals with advanced age may have augmented difficulty with episodic memory, especially when they are required to integrate information from more than one source. ${ }^{11}$ Diminished hippocampal volume ${ }^{13}$ and reduced activity of the middle frontal gyrus are associated with age-related decline in episodic memory retrieval. ${ }^{10}$ Working memory ${ }^{16}$ is known to share a neurocircuitry overlap with attention processes. ${ }^{17}$ Working memory capacity also is closely associated with other cognitive functions, such as shifting and inhibition. ${ }^{10}$ Enhanced cerebellar activity is related to working memory; increased cerebellar activity is likely due to compensatory recruitment of neurons due to reduced activity in the superior frontal gyrus. ${ }^{10}$ The superior frontal gyrus contributes to both working memory as well as executive processing. ${ }^{10}$

Although the cognitive decline associated with aging is inevitable, individuals who experience cognitive decline at an increased rate are predisposed to worse outcomes. One longitudinal cohort study found that adults in their 8th and 9th decades of life with preserved cognitive function had a lower risk of disability and death. ${ }^{18}$

On the other hand, crystallized cognitive functions such as semantic memory,,$^{13}$ shortterm memory, ${ }^{13}$ and emotion regulation ${ }^{16}$ remain largely intact throughout the aging process. Semantic memory, a subtype of episodic memory, is related to associated facts or interpretations of previous occurrences. ${ }^{19}$ This type of memory is detached from an individual's personal experience. ${ }^{20}$ Semantic memory loss classically presents with anomia and detectable lesions in the anterior and temporal lobes. ${ }^{20}$ Emotion regulation deficits are not a part of normal aging; in fact, emotional well-being is known to either improve or remain consistent with age. ${ }^{21}$ Emotional experiences in patients of advanced age may be more complex and unique in comparison to other cognitive abilities. ${ }^{22}$ learning and remembering information. Referred to as "fluid intelligence," these abilities show age-related decline during healthy aging, and even greater decline in individuals with age-related cognitive conditions.

On the other hand, some cognitive abilities do not decline with aging. These include previously acquired knowledge, such as vocabulary and mathematics skills, as well as factual information, such as academic information and the faces of familiar people. These are referred to as "crystallized intelligence," and there is limited evidence that they decline with age. In fact, these abilities do not decline until the moderately severe stage of cortical dementias, and are commonly used to index premorbid cognitive functioning and cognitive reserve.

Why is this distinction between fluid intelligence and crystallized intelligence important? As noted above, many older people do not have early-life experience with technology. Thus, their crystallized intelligence, which is not as vulnerable to decline with aging, does not include information about how to perform many technological tasks. In contrast to today's adolescents and young adults, older adults' academic history typically does not include using smartphones, doing homework via Google Docs, or having homework and classwork assigned via the internet.

Learning how to use new technology requires fluid intelligence, and these abilities are less efficient in older adults. So for many older people, technological tasks can be complex and unfamiliar, and the skills needed to learn how to perform them are also more limited, even in comparison to older adults' own ability when younger. Because many technology-based activities require concurrent performance of multiple tasks, older adults are at a 
disadvantage. ${ }^{4}$ It is not surprising, therefore, that a subset of older adults rate their technology skills as weak, and technology-based tasks as challenging or anxiety-provoking.

However, studies show most older adults' attitudes toward technology remain largely positive, and that they are capable of attaining the necessary skills to use information and communication technology. ${ }^{4,5}$ An individual's perception of his/her age, age-related beliefs, and self-efficacy are associated not only with attitudes toward technology, but possibly with cognition itself. ${ }^{6}$

Education level and socioeconomic factors also influence a person's ability to become proficient in using technology. ${ }^{7-9}$ In fact, socioeconomic factors are more strongly related to access to the internet than age. Many older adults have internet access, but this access does not always translate into full use of its services.

The Box ${ }^{10-22}$ (page 20) describes some of the effects of aging on the brain, and how these changes are reflected in cognitive abilities.

\section{The role of cognitive training}

Existing interventions for helping older adults improve their technology proficiency generally focus on improving cognition, and not necessarily on addressing skills learning. Skills learning and cognition are related; however, the brain depends on neural plasticity for skills learning, whereas cognitive declines are a result of gradual and functional worsening of memory, processing speed, executive functioning, and attention. ${ }^{23}$ Interventions such as cognitive strategy training are capable of altering brain neurocircuitry to improve attention and memory. ${ }^{10,11}$ Other interventions known to improve cognition include exercise ${ }^{10}$ and processing speed training. ${ }^{24}$ On the other hand, skills learning is more effectively targeted by interventions that focus on stimulating realistic environments to mimic activities of daily living that involve technology.

Studies have consistently demonstrated cognitive improvements associated with computerized cognitive training (CCT). The Advanced Cognitive Training for
Independent and Vital Elderly (ACTIVE) study was designed to evaluate the efficacy of cognitive training in 2,832 healthy adults age $>65$ across 6 recruitment sites in the United States. ${ }^{25}$ Participants were randomized to a control group (no treatment) or to 1 of 3 treatment groups:

- memory strategy training (instructorled, not computerized)

- reasoning training (instructor-led, not computerized)

- speed training (no instructor, adaptive computerized training).

Each treatment group received 10 sessions of classroom-based training (1 hour each, twice per week for 5 weeks). Following the intervention, participants who had completed $\geq 8$ sessions were randomized to receive 4 booster sessions at 11 and 35 months after the initial training, or no booster sessions.

Each cognitive training program significantly improved performance on within-domain cognitive tests relative to the control group. Effect sizes were large immediately following training; they declined over time, but were still significant at 10-year follow-up. As hypothesized, training effects did not generalize to neuropsychological tests in other training domains. The booster subgroup of speed training showed improved performance on a separate functional speed measure at 2-year ${ }^{26}$ and 5-year follow-up. ${ }^{27}$ Each condition showed slower decline in instrumental activities of daily living relative to the control group.

The Figure (available at MDedge.com/ psychiatry) shows the type of stimuli presented in the speed training, a procedure where individuals are taught highspeed multitasking by having to identify and locate visual information quickly in a divided-attention format. A stimulus appears in the center of the screen-either a car or a truck-and at the same time, a "Route 66" sign appears in the periphery. For every successful response, the next stimulus is presented at a shorter duration after every successful response, and more slowly after errors.

Secondary outcome analyses demonstrated that for older adults, speed

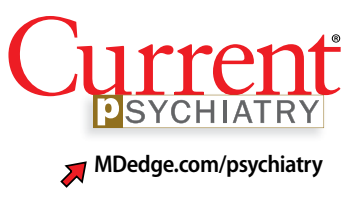

Clinical Point

Skills learning is effectively targeted by interventions that mimic activities of daily living that involve technology 


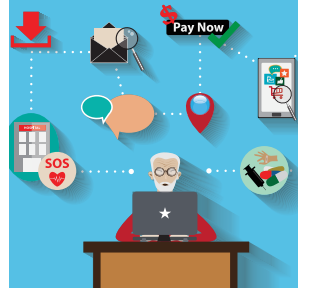

Older adults and technology

\section{Clinical Point}

Concurrent cognitive skills training increased the efficiency of skills training training reduced rates of driving cessation, ${ }^{27}$ improved driving habits, and lowered the incidence of at-fault crashes $^{28}$ (based on motor vehicle records). Speed training also resulted in improvements in health-related quality of life, ${ }^{29,30}$ depression, ${ }^{31}$ locus of control, ${ }^{32}$ and medical expenditures. $^{33}$ An analysis of 10-year outcomes $^{34}$ found that speed training was associated with a $29 \%$ reduction in risk of developing of dementia, while the other 2 interventions were not. However, despite these multiple areas of benefit, there was no evidence that new functional skills were acquired as a result of the training..$^{26-34}$

\section{Functional skills training}

While there is a long history of using functional skills training to help patients with schizophrenia, for healthy older people, there are considerably more challenges. First, aging is not a disease. Consequently, functional skills training is typically not covered by health insurance. Second, functional skills training delivered by a human trainer can be expensive and is not readily available. Finally, there are no real curricula for training functional skills, particularly those that are device-based (phone, tablet, or computer).

Recently, researchers have developed a functional skills assessment and training program that was originally piloted as a fixed difficulty simulation as described in 2 studies by Czaja et al. ${ }^{2,3}$ The original assessment was used to compare healthy control individuals with people with mild cognitive impairment (MCI) or schizophrenia. Most recently, training modules for 6 different technology-based functional tasks have been developed and piloted in samples of healthy controls and patients with $\mathrm{MCI}$ in a randomized trial..$^{35}$ Half of the participants in each of the 2 groups were randomized to receive speed training similar to the ACTIVE study, and the other half received skills training alone. All participants were trained for 24 sessions over 12 weeks or until they mastered all 6 simulations.

Both patients with $\mathrm{MCI}$ and healthy controls improved in all 6 simulations. Although patients with MCI were considerably less efficient at baseline, their training gains per session were equivalent to that of healthy controls. Finally, concurrent cognitive training increased the efficiency of skills training. At the end of the study, functional gains were the same for people in both groups randomized to either condition, even though individuals in the combined cognitive and skills training interventions received only half as much skills training time.

\section{What to tell patients}

Older patients might ask their clinicians what they can do to "exercise their brain." Let them know that CCT has been shown to improve cognitive performance in healthy older people, and that there are several evidence-based, commercially available products for this purpose. Two such selfadministrable systems with supportive data are BrainHQ (www.brainhq.com). and Happy Neuron (www.happy-neuron. com). Explain that it is likely that the best strategy is a combination of cognitive and functional skills training. One commercially available functional skills training program with supportive data is i-Function (www.i-Function.com). (Editor's note: One of the authors, $\mathrm{PDH}$, is an employee of i-Function, Inc.)

\section{References}

1. Harvey PD. Domains of cognition and their assessment. Dialogues Clin Neuro. 2019;21(3):227-237.

2. Czaja SI, Loewenstein DA, Sabbag SA, et al. A novel method for direct assessment of everyday competence among older adults. J Alzheimers Dis. 2017;57(4):1229-1238.

3. Czaja SJ, Loewenstein DA, Lee CC, et al. Assessing functional performance using computer-based simulations of everyday activities. Schizophr Res. 2017;183:130-136.

4. Tsai HS, Shillair R, Cotten SR. Social support and "playing around": an examination of how older adults acquire digital literacy with tablet computers. J Appl Gerontol. 2017;36(1):29-55.

5. Cabrita M, Tabak M, Vollenbroek-Hutten MM. Older adults' attitudes toward ambulatory technology to support monitoring and coaching of healthy behaviors: qualitative study. JMIR Aging. 2019;2(1):e10476. doi: 10.2196/10476.

6. Lim KY, Chang KI, Kim HI, et al. P.5.a.010 association between memory age identity and cognition in the elderly. Eur Neuropsychopharmacol. 2010;20(suppl 3):S555.

7. Moraes C, Pinto JA Jr, Lopes MA, et al. Impact of sociodemographic and health variables on mini-mental state examination in a community-based sample of older people. Eur Arch Psychiatry Clin Neurosci. 2010;260(7):535-542.

8. Freitas S, Simões MR, Alves L, et al. The relevance of sociodemographic and health variables on MMSE normative data. Appl Neuropsychol Adult. 2015;22(4): 311-319.

9. Han C, Jo SA, Jo I, et al. An adaptation of the Korean mini-mental state examination (K-MMSE) in elderly 
Koreans: demographic influence and population-based norms (the AGE study). Arch Gerontol Geriatr. 2008; 47(3):302-310.

10. Yin $\mathrm{S}, \mathrm{Zhu} \mathrm{X}, \mathrm{Li}$ R, et al. Intervention-induced enhancement in intrinsic brain activity in healthy older adults. Sci Rep. 2014;4:7309.

11. Bender AR, Prindle JJ, Brandmaier AM, et al. White matter and memory in healthy adults: coupled changes over two years. Neuroimage. 2016;131:193-204.

12. Guye $S$, von Bastian CC. Working memory training in older adults: Bayesian evidence supporting the absence of transfer. Psychol Aging. 2017;32(8):732-746.

13. Taki Y, Kinomura S, Sato K, et al. Correlation between gray/ white matter volume and cognition in healthy elderly people. Brain Cogn. 2011;75(2):170-176.

14. Cassidy AR, White MT, DeMaso DR, et al. Processing speed, executive function, and academic achievement in children with dextro-transposition of the great arteries: Testing a longitudinal developmental cascade model. Neuropsychology. 2016;30(7):874-885

15. Aichele S, Rabbitt P, Ghisletta P. Life span decrements in fluid intelligence and processing speed predict mortality risk. Psychol Aging. 2015;30(3):598-612.

16. Eich TS, Castel AD. The cognitive control of emotional versus value-based information in younger and older adults. Psychol Aging. 2016;31(5):503-512.

17. Rolle CE, Anguera JA, Skinner SN, et al. Enhancing spatial attention and working memory in younger and older adults. J Cogn Neurosci. 2017;29(9):1483-1497.

18. Yaffe $K$, Lindquist $K$, Vittinghoff $E$, et al. The effect of maintaining cognition on risk of disability and death. J Am Geriatr Soc. 2010;58(5):889-894.

19. Madore KP, Schacter DL. An episodic specificity induction enhances means-end problem solving in young and older adults. Psychol Aging. 2014;29(4):913-924.

20. Matthews BR. Memory dysfunction. Continuum (Minneap Minn). 2015;21(3 Behavioral Neurology and Neuropsychiatry):613-626.

21. Mather M. The emotion paradox in the aging brain. Ann N Y Acad Sci. 2012;1251(1):33-49.

22. Gurera JW, Isaacowitz DM. Emotion regulation and emotion perception in aging: A perspective on age-related differences and similarities. Prog Brain Res. 2019;247:329-351.

23. Cai L, Chan JS, Yan JH, et al. Brain plasticity and motor practice in cognitive aging. Front Aging Neurosci. 2014;6:31.

24. Cassetta BD, Tomfohr-Madsen LM, Goghari VM. A randomized controlled trial of working memory and processing speed training in schizophrenia. Psychol Med. 2019;49(12):2009-2019.

25. Ball K, Berch DB, Helmers KF, et al. Effects of cognitive training interventions with older adults: a randomized controlled trial. JAMA. 2002;288(18):2271-2281.

\section{Related Resources}

- Hill NT, Mowszowski L, Naismith SL, et al. Computerized cognitive training in older adults with mild cognitive impairment or dementia: a systematic review and metaanalysis. Am J Psychiatry. 2017;174(4):329-340.

- Harvey PD, McGurk SR, Mahncke H, et al. Controversies in computerized cognitive training. Biol Psychiatry Cogn Neurosci Neuroimaging. 2018;3(11):907-915.

26. Rebok GW, Ball K, Guey LT, et al. Ten-year effects of the advanced cognitive training for independent and vital elderly cognitive training trial on cognition and everyday functioning in older adults. J Am Geriatr Soc. 2014;62(1):16-24.

27. Edwards JD, Delahunt PB, Mahncke HW. Cognitive speed of processing training delays driving cessation. J Gerontol A Biol Sci Med Sci. 2009;64(12):1262-1267.

28. Ball K, Edwards JD, Ross LA, et al. Cognitive training decreases motor vehicle collision involvement of older drivers. J Am Geriatr Soc. 2010;58(11):2107-2113.

29. Wolinsky FD, Unverzagt FW, Smith DM, et al. The effects of the ACTIVE cognitive training trial on clinically relevant declines in health-related quality of life. J Gerontol B Psychol Sci Soc Sci. 2006;61(5):S281-S287.

30. Wolinsky FD, Unverzagt FW, Smith DM, et al. The ACTIVE cognitive training trial and health-related quality of life: protection that lasts for 5 years. J Gerontol A Biol Sci Med Sci. 2006;61(12):1324-1329.

31. Wolinsky FD, Vander Weg MW, Martin R, et al. The effect of speed-of-processing training on depressive symptoms in ACTIVE. J Gerontol A Biol Sci Med Sci. 2009;64(4): 468-472.

32. Wolinsky FD, Vander Weg MW, Martin R, et al. Does cognitive training improve internal locus of control among older adults? J Gerontol B Psychol Sci Soc Sci. 2010;65(5): 591-598.

33. Wolinsky FD, Mahncke HW, Kosinski M, et al. The ACTIVE cognitive training trial and predicted medical expenditures. BMC Health Serv Res. 2009;9:109.

34. Edwards JD, Xu H, Clark DO, et al. Speed of processing training results in lower risk of dementia. Alzheimers Dement (N Y). 2017;3(4):603-611.

35. Harvey PD, Tibiriçá L, Kallestrup P, et al. A computerized functional skills assessment and training program targeting technology based everyday functional skills. J Vis Exp. 2020;156:e60330. doi: 10.3791/60330.

\section{Bottom Line}

Clinicians should ensure older patients that they have the cognitive capacity to learn new technology-related functional skills, and that such patients have the opportunity to learn these skills. Clinicians need to be able to identify people who are at high risk of not being able to adhere to instructions and suggestions that require interactions with technology. Treatment options include computerized cognitive training and functional skills training. 


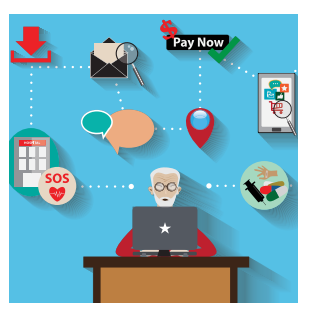

Older adults and technology
Figure

Example of speed training from the ACTIVE study

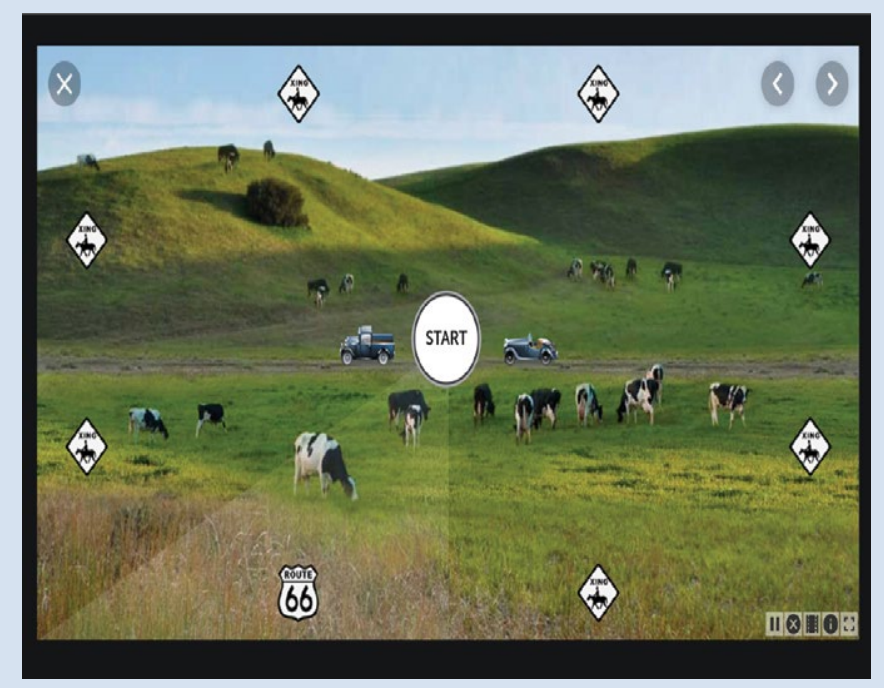

ACTIVE: Advanced Cognitive Training for Independent and Vital Elderly 